\title{
Théorème des trois longueurs et suites sturmiennes : mots d'agencement des longueurs
}

\author{
par \\ Anne Siegel (Marseille)
}

0. Introduction. Le théorème des trois longueurs (voir le survol [1]) affirme que si $\alpha$ est irrationnel et $N$ entier, les points $\{k \alpha\}$, pour $0 \leq k<N$, partitionnent le cercle de périmètre 1 en arcs dont la longueur ne prend que deux ou trois valeurs. Lorsque $N=q_{n}+i q_{n-1}$, pour $i \leq a_{n+1}$, est le dénominateur d'un convergent partiel de $\alpha$, seules deux longueurs apparaissent sur le cercle. On peut alors définir un mot sur l'alphabet $\{0,1\}$ qui décrit l'ordre d'apparition des deux longueurs possibles. Nous étudions les mots définis lorsque $N$ est de la forme $N=q_{n}+q_{n-1}$, les mots obtenus pour les autres convergents pouvant se déduire de ceux-là.

DÉfinition. Pour tout $n$ non nul, $\Psi_{n}$ est le mot de $N=q_{n}+q_{n-1}$ lettres sur l'alphabet $\{0,1\}$ dont la $j$-ème lettre est 1 si et seulement si le $j$-ème arc apparaissant sur le cercle de périmètre 1 subdivisé par les points $0,\{\alpha\}$, $\ldots,\{(N-1) \alpha\}$ est de longueur $(-1)^{n-1}\left(q_{n-1} \alpha-p_{n-1}\right)$. Les mots $\Psi_{n}$ sont appelés mots d'agencement des longueurs.

Une étude plus approfondie [14] des nombres $\{n \alpha\}$ montre que chaque mot $\Psi_{n}$ est l'image du précédent par une substitution appartenant au monoïde de Sturm - étudié dans le cadre de la caractérisation des mots sturmiens points fixes de substitutions (voir [7, 11]). Ces substitutions préservent la propriété combinatoire d'équilibre, c'est-à-dire le fait que les nombres d'occurrences de la lettre 0 dans deux facteurs de même longueur diffèrent au plus de 1 .

THÉORÈME. Les mots d'agencement des longueurs sont équilibrés et leurs valeurs d'adhérence sont des mots soit sturmiens - c'est-à-dire qu'il ont pour tout entier $n$ exactement $n+1$ facteurs de longueur $n-$ soit ultimement périodiques.

2000 Mathematics Subject Classification: Primary 11J71; Secondary 68R15, 11J70, $11 \mathrm{~B} 95$. 
Nous utilisons dans cet article le lien étroit qui existe entre les valeurs d'adhérence des mots d'agencement des longueurs et les valeurs d'adhérence des mots initiaux de la suite des quotients partiels lus à l'envers, notés $a_{n} \ldots a_{1}$.

En effet, l'étude de [14] implique que les mots d'agencement des longueurs sont gouvernés par ces mots lus à l'envers. On en déduit au théorème 2.1 que les valeurs d'adhérence ultimement périodiques des mots d'agencement sont obtenues à partir des sous-suites de quotients partiels de $\alpha$ tendant vers l'infini. Ceci implique des résultats du type :

Proposition. Les valeurs d'adhérence de la suite des mots d'agencement des longueurs sont toutes sturmiennes si et seulement si les quotients partiels du développement en fraction continue de $\alpha$ sont bornés.

On montre avec un raisonnement similaire que les mots infinis de $\mathbb{N}^{\mathbb{N}}$ qui sont valeurs d'adhérence de la suite $a_{n} \ldots a_{1}$ des quotients partiels lus à l'envers, sont en bijection avec les valeurs d'adhérence sturmiennes de la suite des mots d'agencement des longueurs (proposition 3.1). Ceci permet d'identifier les valeurs d'adhérence sturmiennes en appliquant à des codages de rotations des techniques d'induction utilisées dans l'étude des échanges d'intervalles (voir par exemple $[2,10]$ ).

THÉORÈME. Les valeurs d'adhérence sturmiennes des mots d'agencement des longueurs associés à un angle $\alpha$ de convergents $p_{n} / q_{n}$ et de quotients partiels $a_{n}$ sont les codages de la trajectoire de $1-\beta$ par la rotation d'angle $\beta$ sur $[0,1[$, par rapport à la partition $[0,1-\beta[\cup[1-\beta, 1[$ (respectivement $] 0,1-\beta] \cup] 1-\beta, 1]$ ), ceci pour toutes les valeurs d'adhérence irrationnelle $\beta$ de la suite de nombres $\left[0 ; 1, a_{n}, \ldots, a_{1}\right]=q_{2 n} /\left(q_{2 n}+q_{2 n-1}\right)$ (respectivement $\left.q_{2 n+1} /\left(q_{2 n}+q_{2 n+1}\right)\right)$.

Le plus grand angle des valeurs d'adhérence des mots d'agencement est ainsi fortement lié au quotient de récurrence des suites sturmiennes d'angle $\alpha$, calculé dans [6]. De plus, l'ergodicité de l'opérateur de décalage sur les fractions continues implique que :

Corollaire. Pour presque tout angle $\alpha$, tout élément de l'ensemble St des facteurs des mots sturmiens est facteur d'un mot d'agencement des longueurs.

Cet article est organisé comme suit : on définit dans le paragraphe 1 les mots d'agencement des longueurs. On étudie dans le paragraphe 2 les valeurs d'adhérence ultimement périodiques de ces mots, et leurs valeurs d'adhérence sturmiennes dans le paragraphe 3. Dans le paragraphe 4, on s'intéresse au cas quadratique et on montre que les valeurs d'adhérence sont alors toutes sturmiennes, en nombre fini, et points fixes de substitutions qu'on détermine. Enfin, dans le paragraphe 5, on généralise les résultats 
précédents aux mots d'agencement des longueurs définis pour n'importe quel convergent partiel.

Remerciements. Je remercie V. Berthé et P. Arnoux pour leurs précieux conseils, patience et soutien. Je remercie également le rapporteur de cet article et J.-P. Allouche pour leurs commentaires judicieux.

1. Mots d'agencement des longueurs. Pour tout réel $x$, on note $[x]$ sa partie entière et $\{x\}$ sa partie fractionnaire. Dans tout ce qui suit, $\alpha$ est un irrationnel de ]0,1[.

Le théorème des trois longueurs, ou conjecture de Steinhaus, a été prouvé par S. Świerczkowski [13]. De nouvelles preuves ont ensuite été données par de nombreux auteurs (voir $[1,12,14]$ ).

Ce théorème a un lien profond avec la théorie des fractions continues. Rappelons que si $\alpha$ est irrationnel, le développement en fraction continue de $\alpha$ est obtenu (voir [8]) par l'algorithme qui définit, pour tout $n$, les nombres $a_{n}$ et $t_{n}$ :

$$
t_{0}=\alpha, \quad a_{n}=\left[t_{n}\right] \quad \text { et } t_{n+1}=1 /\left\{t_{n}\right\} .
$$

Les entiers $a_{n}$ sont appelés quotients partiels. Du fait de leur définition, ils vérifient la formule

$$
\alpha=a_{0}+\frac{1}{a_{1}+\frac{1}{\ddots+\frac{1}{a_{n-1}+\frac{1}{t_{n}}}}}=\left[a_{0} ; a_{1}, \ldots, a_{n-1}, t_{n}\right] .
$$

On appelle convergents partiels de $\alpha$ les rationnels suivants, mis sous leur forme irréductible $: p_{n, i} / q_{n, i}=\left[a_{0} ; a_{1}, \ldots, a_{n-1}, i\right]$. Les convergents totaux sont obtenus pour $i=a_{n}$ (on note $p_{n}=p_{n, a_{n}}$ et $\left.q_{n}=q_{n, a_{n}}\right)$. On a alors $p_{n, i}=i p_{n-1}+p_{n-2}$ et $q_{n, i}=i q_{n-1}+q_{n-2}$.

Les convergents partiels sont en fait les nombres de Farey associé à l'angle $\alpha$ : chaque $p_{n, i} / q_{n, i}$ est le rationnel qui approxime le mieux l'angle $\alpha$, parmi les rationnels de dénominateur strictement inférieur à $q_{n, i+1}$.

Par un abus de langage, on appelle les dénominateurs $q_{n, i}$ convergents partiels associés à $\alpha$. Le théorème des trois longueurs s'énonce comme suit.

ThÉORÈme DES TROIS LONGUEURS. Si $\alpha$ est irrationnel et $N$ entier, les points $\{i \alpha\}$, pour $0 \leq i \leq N-1$, partitionnent le cercle de périmètre 1 en $N$ arcs dont la longueur ne prend que deux ou trois valeurs, l'une étant la somme des deux autres.

Plus précisément, si $q_{k+1, i}$ est le plus grand convergent partiel associé à $\alpha$ qui minore $N$ strictement, c'est-à-dire $N=q_{k+1, i}+r$, avec $0<r \leq q_{k}$, alors les points $0,\{\alpha\}, \ldots,\{(N-1) \alpha\}$ partitionnent le cercle en $N$ intervalles : 
- $\left(N-q_{k}\right)$ sont de longueur $(-1)^{k}\left(q_{k} \alpha-p_{k}\right)$,

- $r$ sont de longueur $(-1)^{k-1}\left(q_{k-1} \alpha-p_{k-1}\right)-i(-1)^{k}\left(q_{k} \alpha-p_{k}\right)$,

- $\left(q_{k}-r\right)$ sont de longueur $(-1)^{k}\left(-\left(q_{k-1} \alpha-p_{k-1}\right)+(i-1)\left(q_{k} \alpha-p_{k}\right)\right)$.

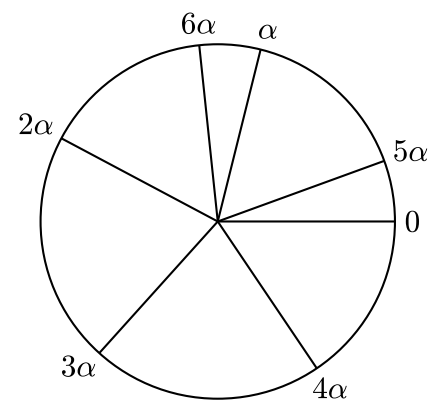

Fig. 1. Illustration du théorème des trois longueurs

En particulier, si $r=q_{k}$, c'est-à-dire si $N$ est un convergent partiel, seules deux longueurs apparaissent, et le cercle orienté subdivisé fournit un mot fini sur l'alphabet $\{0,1\}$, correspondant à l'ordre d'apparition des longueurs. On s'intéresse aux mots obtenus pour les entiers de la forme $N=q_{n+1,1}=q_{n}+q_{n-1}$. Le premier point qui n'a pas encore été placé sur le cercle est $\left\{q_{n+1,1} \alpha\right\}$.

DÉfinition 1.1. Pour tout $n$ non nul, $\Psi_{n}$ est le mot de $N=q_{n}+q_{n-1}$ lettres sur l'alphabet $\{0,1\}$ dont la $j$-ème lettre est 1 si et seulement si le $j$-ème arc apparaissant sur le cercle subdivisé par les points $0,\{\alpha\}, \ldots$ et $\{(N-1) \alpha\}$ est de longueur $(-1)^{n-1}\left(q_{n-1} \alpha-p_{n-1}\right)$.

On pose $\Psi_{0}=1$. Les mots $\Psi_{n}$ ainsi définis sont appelés mots d'agencement des longueurs ou plus simplement mots d'agencement.

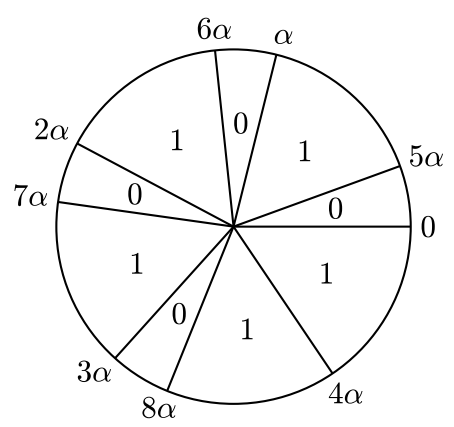

Mot d'agencement : 010101011

Fig. 2. Un exemple de mot d'agencement des longueurs 
Une analyse plus fine de la manière dont s'insèrent les points $\left\{q_{n+1, i} \alpha\right\}$ sur le cercle implique que cette suite de mots est liée aux substitutions suivantes - une substitution est un endomorphisme de monoïde libre pour la concaténation.

DÉfinition 1.2. Pour tout $n$, on appelle $I_{n}$ et $J_{n}$ les substitutions suivantes :

$$
\begin{array}{ll}
I_{n}(0)=1, & J_{n}(0)=1, \\
I_{n}(1)=01^{n}, & J_{n}(1)=1^{n} 0 .
\end{array}
$$

Proposition 1.1 (voir [14]). Si les $a_{n}$ sont les quotients partiels d'un irrationnel $\alpha$, les mots d'agencement des longueurs $\Psi_{n}$ associés à $\alpha$ vérifient la formule

$$
\forall n>0 \quad \Psi_{n}= \begin{cases}I_{a_{n}}\left(\Psi_{n-1}\right) & \text { si } n \text { est pair } \\ I_{a_{2 n+1}}\left(\Psi_{n-1}\right) & \text { si } n \text { est impair. }\end{cases}
$$

Du fait de leur définition, les substitutions $I_{n}$ et $J_{n}$ appartiennent toutes au monö̈de de Sturm, c'est-à-dire l'ensemble des substitutions engendrées par $\sigma_{1}, \sigma_{2}$ et $E$ :

$$
\begin{array}{lll}
E(0)=1, & \sigma_{1}(0)=0, & \sigma_{2}(0)=0, \\
E(1)=0, & \sigma_{1}(1)=01, & \sigma_{2}(1)=10 .
\end{array}
$$

Ce monoïde, qui contient en particulier la substitution $\sigma_{1} E(0 \mapsto 01$, $1 \mapsto 0$ ), plus connue sous le nom de morphisme de Fibonacci, a été étudié dans le cadre de la caractérisation des mots sturmiens points fixes de substitutions (voir par exemple $[7,11]$ ). Selon un raisonnement classique que nous reprenons ci-dessous, ses éléments conservent la propriété combinatoire d'équilibre. On rappelle qu'un mot infini équilibré est tel que la différence des nombres d'occurrences d'une lettre dans deux de ses facteurs de même longueur est toujours bornée par 1 en valeur absolue, et qu'un mot fini équilibré est un facteur d'un mot infini équilibré.

Lemme 1.1. Les mots d'agencement des longueurs $\Psi_{n}$ sont équilibrés.

Preuve. Le mot $\Psi_{0}=1$ étant équilibré, il est suffisant de montrer que les trois générateurs du monoïde de Sturm conservent l'équilibre des mots. Par symétrie, on est amené à montrer ceci pour $\sigma_{1}$.

Si l'image d'un mot $U$ équilibré n'est pas équilibrée, il existe deux facteurs de $\sigma_{1}(U)$ de même longueur, dont la différence des nombres d'occurrences de 0 n'est pas bornée par 1 . On prouve ainsi l'existence d'un facteur $W$ tel que $0 W 0$ et $1 W 1$ apparaissent dans $\sigma_{1}(U)$.

La lettre 1 étant isolée dans $\sigma_{1}(U)$, le mot $W$ s'écrit $W=0 Z 0$, et $\sigma_{1}(U)$ admet pour facteurs les deux mots $W_{1}=00 Z 00$ et $W_{2}=10 Z 010$. Mais le deuxième et le dernier 0 de $W_{1}$ ne peuvent provenir par $R$ que de la lettre 0 , 
ce qui implique que $Z 0=\sigma_{1}(V), V$ facteur de $U$. On obtient ainsi que $\sigma_{1}(0 V 0)$ et $\sigma_{1}(1 V 1)$ apparaissent dans $\sigma_{1}(U)$, et $0 V 0$ et $1 V 1$ dans $U$, ce qui est impossible.

Les mots infinis équilibrés se divisent en deux catégories. Un mot infini est dit sturmien s'il contient exactement $n+1$ facteurs distincts de longueur $n$ pour tout entier $n$, c'est-à-dire qu'il est de complexité minimale parmi les mots non ultimement périodiques. Dans [9], Morse et Hedlund montrent que les mots sturmiens sont en fait les mots infinis équilibrés non ultimement périodiques. Ils prouvent aussi qu'un mot infini $u$ est sturmien si et seulement si c'est un codage de rotation, c'est-à-dire si et seulement s'il existe un irrationnel $\beta \in] 0,1[$ et un nombre $\varrho$ - appelé intercept - tel que $u$ code la trajectoire sur $] 0,1[$ de $\varrho$ par la rotation d'angle $\beta$ sur les intervalles ] $0,1-\beta]$ et $] 1-\beta, 1]$ (on parle alors de codage à gauche), ou sur les intervalles $[0,1-\beta[$ et $[1-\beta, 1[$ (codage à droite). Le mot caractéristique d'angle $\beta$ est le mot sturmien d'intercept nul. C'est le seul mot sturmien d'angle $\beta$ qui admet deux extensions gauches sturmiennes : les codages à droite et à gauche d'intercept $1-\beta$.

L'équivalence entre ces définitions arithmétique et combinatoire a inspiré de nombreuses applications de ces mots sturmiens, entre autres exemples en cristallographie, en infographie et en théorie ergodique (voir [3] pour une synthèse des travaux). En particulier, la définition géométrique des mots sturmiens implique que le théorème des trois longueurs a une formulation liée aux mots sturmiens : dans un mot d'angle $\beta$, les fréquences des facteurs de longueur $m$ ne peuvent prendre que trois valeurs, qui sont les trois longueurs obtenues en plaçant sur le cercle les points $(k\{1-\beta\})_{0 \leq k<m}$ (voir [4]).

Dans cet article, on explore un lien plus subtil entre le théorème des trois longueurs et les mots sturmiens. En effet, le lemme 1.1 signifie que les mots d'agencement des longueurs sont des facteurs de mots infinis équilibrés, c'est-à-dire sturmiens ou ultimement périodiques. Nous allons chercher à identifier ces mots équilibrés, en s'intéressant aux valeurs d'adhérence des mots d'agencement. Ainsi, le lemme 1.1 implique directement le théorème suivant. On rappelle que l'ensemble des mots finis et infinis sur l'alphabet $\{0,1\}$ est un espace compact pour la topologie discrète. Selon cette topologie, deux mots sont d'autant plus proches qu'ils ont même début. Elle est aussi définie par la métrique suivante, où $|u| \in[0,+\infty]$ est la longueur du mot $u$ :

$$
d\left(\left(u_{n}\right)_{0 \leq n<|u|},\left(v_{n}\right)_{0 \leq n<|v|}\right)=\left\{\begin{array}{l}
0 \quad \operatorname{si}\left(u_{n}\right)_{0 \leq n<|u|}=\left(v_{n}\right)_{0 \leq n<|v|}, \\
\exp \left(-\min \left\{n, u_{n} \neq v_{n},|u|,|v|\right\}\right)
\end{array}\right. \text { sinon. }
$$

ThÉorÈme 1.1. Les valeurs d'adhérence de la suite des mots d'agencement des longueurs $\left(\Psi_{n}\right)_{n>0}$ associés à un irrationnel $\alpha$ sont des mots infinis ultimement périodiques ou sturmiens. 
2. Valeurs d'adhérence ultimement périodiques. Grâce aux règles de transformation de la formule (1), on détermine le début des mots d'agencement $\Psi_{n}$ associé à $\alpha$ en fonction des quotients partiels $a_{n}$ de $\alpha$ :

$$
\begin{aligned}
& \Psi_{0}=1, \\
& \Psi_{1}=1^{a_{1}} 0, \\
& \Psi_{2 n} \text { commence par } 01^{a_{2 n}} 0 \text { si } n>0, \\
& \Psi_{2 n+1} \text { commence par } 1^{1+a_{2 n+1}} 0 \text { si } n>1 .
\end{aligned}
$$

Lemme 2.1. Une suite extraite $\left(\Psi_{k(n)}\right)$ de la suite des mots d'agencement des longueurs converge vers $1^{\infty}$ ou $01^{\infty}$ si et seulement si d'une part, $k(n)$ est de parité constante à partir d'un certain rang, et d'autre part, les quotients partiels $a_{k(n)}$ tendent vers l'infini.

Preuve. Si les mots d'agencement $\Psi_{k(n)}$ convergent vers un mot $u$, la première lettre de $u$ est la première lettre des $\Psi_{k(n)}$ à partir d'un certain rang, ce qui implique (formules (2)) que $k(n)$ est de parité constante, par exemple impaire. Si les quotients partiels $a_{k(n)}$ ne tendent pas vers l'infini, ils admettent une sous-suite bornée, dont on peut extraire une sous-suite $\left(a_{m(n)}\right)$ constante égale à $a$. Les mots $\Psi_{m(n)}$ commencent ainsi par $1^{1+a} 0$ et ne peuvent converger vers $1^{\infty}$ ou $01^{\infty}$. Les formules (2) impliquent directement la réciproque.

Corollaire 2.1. Les mots $1^{\infty}$ et $01^{\infty}$ sont les seules valeurs d'adhérence de la suite des mots d'agencement des longueurs associés à $\alpha$ si et seulement si les quotients partiels de $\alpha$ tendent vers l'infini.

Preuve. Si les quotients partiels $a_{n}$ ne tendent pas vers l'infini, il existe une suite extraite $a_{k(n)}$ qui est bornée. Par compacité, les mots $\Psi_{k(n)}$ admettent une sous-suite convergente $\Psi_{m(n)}$. Sa limite est une valeur d'adhérence qui ne peut-être égale à $1^{\infty}$ ou $01^{\infty}$, sans quoi $m(n)$ tendrait vers l'infini. La réciproque est immédiate.

Le lemme 2.1 permet de caractériser les valeurs d'adhérence ultimement périodiques des mots $\Psi_{n}$ :

THÉORÈme 2.1. La suite des mots d'agencement des longueurs $\left(\Psi_{n}\right)$ associée à l'angle a admet un mot ultimement périodique u pour valeur d'adhérence si et seulement s'il existe une suite d'entiers $k(n)$ de parité constante, et un entier $p$ qui vérifient les conditions suivantes:

- les quotients partiels $a_{k(n)}$ tendent vers l'infini,

- les $p$ suites d'entiers $\left(a_{k(n)+1}\right) \ldots\left(a_{k(n)+p}\right)$ sont constantes égales à des entiers $b_{1} \ldots b_{p}$, 


$$
u= \begin{cases}I_{b_{p}} J_{b_{p-1}} \ldots J_{b_{1}}\left(01^{\infty}\right) & \text { si } k(n) \text { est pair et } p \text { pair } \\ J_{b_{p}} I_{b_{p-1}} \ldots J_{b_{1}}\left(01^{\infty}\right) & \text { si } k(n) \text { est pair et } p \text { impair } \\ J_{b_{p}} I_{b_{p-1}} \ldots I_{b_{1}}\left(1^{\infty}\right) & \text { si } k(n) \text { est impair et } p \text { pair } \\ I_{b_{p}} J_{b_{p-1}} \ldots I_{b_{1}}\left(1^{\infty}\right) & \text { si } k(n) \text { est impair et } p \text { impair. }\end{cases}
$$

Preuve. Si les conditions sont vérifiées, la suite $\left(\Psi_{k(n)}\right)$ converge selon le lemme 2.1 vers $v \in\left\{01^{\infty}, 1^{\infty}\right\}$, et la substitution $Q$ de l'enoncé est selon la formule (1) telle que $Q\left(\Psi_{k(n)}\right)=\Psi_{k(n)+p}$ dès que $a_{k(n)+i}=b_{i}$ pour $i \leq p$, ce qui est vrai pour $n$ assez grand. Ainsi, $u=Q(v)$ est la limite des $\Psi_{k(n)+p}$.

Réciproquement, si $u$ est valeur d'adhérence des $\Psi_{n}$, les mots $\Psi_{k(n)}$ dont elle est la limite commencent par la même lettre à partir d'un certain rang et les formules (2) impliquent, quitte à restreindre la suite $k(n)$, que la parité de $k(n)$ est constante, par exemple paire. Si $u \in\left\{01^{\infty}, 1^{\infty}\right\}$, le lemme 2.1 implique que les quotients partiels $a_{k(n)}$ tendent vers l'infini, et les hypothèses sont vérifiées avec $p=0$. Dans le cas contraire, $u$ commence par $\gamma 1^{b} 0$ avec $\gamma \in\{0,1\}$, et à partir d'un certain rang, les quotients $a_{k(n)}$ sont tous égaux à $b$ (formules $(2)$ ). Ainsi, les $I_{k(n)}$ sont toutes égales à une même substitution $Q$.

Par compacité, on peut supposer que les mots $\Psi_{k(n)-1}$ convergent eux aussi vers un mot $v$, qui va vérifier $Q(v)=u$. Les substitutions $\sigma_{1}$ et $\sigma_{2}$ sont telles que la préimage par ces applications d'un mot ultimement périodique est un mot ultimement périodique de période strictement inférieure, sauf si les deux mots sont ultimement constants (voir [11]). La substitution $Q$ étant engendré par $\sigma_{1}$ et $\sigma_{2}$, on en déduit que $v$ est ultimement périodique, de période strictement inférieure à celle de $u$. De proche en proche, on exhibe ainsi une succession de mots ultimement périodiques dont la période décroît strictement. Ce processus s'arrête après un nombre fini $p$ d'étapes, et le mot obtenu est alors nécessairement $1^{\infty}$ ou $01^{\infty}$. Privée de ses premiers termes, la suite $(k(n)+p)_{k>0}$ vérifie les conditions requises.

Corollaire 2.2. Si les mots d'agencement des longueurs admettent des valeurs d'adhérence ultimement périodiques, un des deux mots $1^{\infty}$ ou $01^{\infty}$ est lui-même valeur d'adhérence.

Preuve. Si les mots d'agencement ont une valeur d'adhérence ultimement périodique, alors on vient de montrer qu'une sous-suite de quotients partiels tend vers l'infini, ce qui implique selon le lemme 2.1 que $1^{\infty}$ ou $01^{\infty}$ est valeur d'adhérence.

On caractérise la situation inverse grâce à ce corollaire et au lemme 2.1.

COROLlaire 2.3. Les valeurs d'adhérence des mots d'agencement des longueurs associés à un angle $\alpha$ sont toutes sturmiennes si et seulement si les quotients partiels de $\alpha$ sont bornés. 
3. Valeurs d'adhérence sturmiennes. Avec un raisonnement proche de celui du théorème 2.1, on relie les valeurs d'adhérences sturmiennes des mots d'agencement $\Psi_{n}$ aux quotients partiels. L'ensemble $\bigcup_{n>0} \mathbb{N}^{n} \cup \mathbb{N}^{\mathbb{N}}$ est muni de la topologie discrète, qui est aussi définie par la métrique suivante :

$$
d\left(\left(u_{n}\right)_{0 \leq n<|u|},\left(v_{n}\right)_{0 \leq n<|v|}\right)=\left\{\begin{array}{l}
0 \quad \text { si }\left(u_{n}\right)_{0 \leq n<|u|}=\left(v_{n}\right)_{0 \leq n<|v|}, \\
\exp \left(-\min \left\{n, u_{n} \neq v_{n},|u|,|v|\right\}\right)
\end{array}\right. \text { sinon. }
$$

Proposition 3.1. L'application

$$
\begin{gathered}
\left(b_{n}\right)_{n \geq 0} \in \mathbb{N}^{\mathbb{N}} \rightarrow \lim _{n \rightarrow \infty} I_{b_{1}} J_{b_{2}} \ldots J_{b_{2 n}}(0) \in\{0,1\}^{\mathbb{N}} \\
\left(\text { respectivement }\left(b_{n}\right)_{n \geq 0} \rightarrow \lim _{n \rightarrow \infty} J_{b_{1}} I_{b_{2}} \ldots J_{b_{2 n+1}}(0)\right)
\end{gathered}
$$

met en bijection l'ensemble des valeurs d'adhérence de la suite de mots $\left(a_{2 n} a_{2 n-1} \ldots a_{1}\right)$ (respectivement $\left.\left(a_{2 n+1} a_{2 n} \ldots a_{1}\right)\right)$ et l'ensemble des valeurs d'adhérence sturmiennes débutant par 0 (respectivement par 1) de la suite des mots d'agencement des longueurs.

Preuve. Soit $u$ une valeur d'adhérence des mots $\Psi_{n}$, sturmienne et commençant par 0. Les formules (2) impliquent que $u$ est limite d'une soussuite de la forme $\left(\Psi_{2 k(n)}\right)$. De plus, $u$ est un mot sturmien et est donc distinct de $01^{\infty}$. Soit $b_{1}$ la plus grande puissance de 1 apparaissant dans le début de $u$ privé de son premier 0 . Selon les formules (2), les quotients $a_{2 k(n)}$ sont alors égaux à $b_{1}$ à partir d'un certain rang. Le fait que les mots $\Psi_{2 k(n)}=I_{b_{1}}\left(\Psi_{2 k(n)-1}\right)$ convergent tandis que $Q$ est injective sur les lettres implique que la suite $\left(\Psi_{2 k(n)-1}\right)$ ne peut que converger vers un mot $v$ qui vérifie $I_{b_{1}}(v)=u$ et n'est donc pas ultimement périodique - sans quoi $u$ le serait aussi (voir [11]). De proche en proche, on en déduit que chaque suite $\left(a_{2 k(n)-p}\right)$, pour $p$ fixé, vaut une constante $b_{p}$ à partir d'un certain rang, ce qui équivaut à dire que les mots $a_{2 k(n)} a_{2 k(n)-1} \ldots a_{1}$ convergent vers le mot $\left(b_{n}\right)$, qui est entièrement déterminé par $u$ et vérifie que $u=\lim \Psi_{2 k(n)}=\lim I_{b_{1}} J_{b_{2}} \ldots J_{b_{2 k(n)}}(0)$.

Réciproquement, si une sous-suite $\left(a_{2 k(n)} a_{2 k(n)-1} \ldots a_{1}\right)$ converge vers un mot infini $\left(b_{n}\right)$, chaque suite $\left(a_{2 k(n)-p}\right)$, à $p$ fixé, va être égale à $b_{p}$ à partir d'un certain rang. Ceci permet de définir pour tout $p$ une substitution $S_{p}$ (égale à $I_{b_{p}}$ ou $J_{b_{p}}$ ) et un entier $n(p)$ tels que $Q_{2 k(n)-p}=S_{p}$ pour $n>n(p)$. Les mots $S_{1} S_{2} \ldots S_{2 p-1}(0)$ sont débuts les uns des autres et convergent donc vers un mot $u$. Chacun de ces mots est le début de $\Psi_{2 k(n)}$ dès que $n>n(p)$. On en déduit que les $\Psi_{2 k(n)}$ convergent vers $u$, et que $u$ ne peut pas être ultimement périodique, sans quoi une sous-suite $\left(a_{2 k(n)-p}\right)_{n>0}$ tendrait vers l'infini (théorème 2.1) et ne pourrait être constante à partir d'un certain rang. La preuve pour les valeurs d'adhérence débutant par 1 est similaire.

On en déduit une condition nécessaire et non suffisante sur $\alpha$ pour l'existence d'une valeur d'adhérence sturmienne. On dit qu'une sous-suite 
$\left(x_{k(n)}\right)$ d'une suite $\left(x_{n}\right)$ est à lacunes bornées s'il existe une constante $M$ telle que $k(n+1)-k(n)<M$ pour tout $n$.

Corollaire 3.1. Si la suite des mots d'agencement des longueurs associée à un angle $\alpha$ admet une valeur d'adhérence sturmienne, alors la suite des quotients partiels de $\alpha$ n'admet aucune sous-suite à lacunes bornées qui tend vers l'infini.

La réciproque de cette affirmation est fausse, comme le montre l'exemple de l'angle $\alpha$ dont les quotients partiels sont définis comme suit:

$$
a_{n^{2}+i}= \begin{cases}n & \text { si } i=0 \\ i & \text { si } 0<i<2 n+1\end{cases}
$$

Preuve. Si les mots d'agencement admettent une valeur d'adhérence sturmienne, une sous-suite $\left(a_{k(n)} a_{k(n)-1} \ldots a_{1}\right)$ converge, et, $p$ étant fixé, tous les quotients partiels $a_{k(n)}, a_{k(n)-1}, \ldots, a_{k(n)-p}$ sont bornés. Ainsi, la suite $\left(a_{n}\right)$ ne peut admettre de sous-suite à lacune bornée par $p$ qui tend vers l'infini.

Si, pour l'angle $\alpha$ défini en énoncé, les mots d'agencement ont une valeur d'adhérence sturmienne, il existe une suite d'indice $k(n)$ telle que les $a_{k(n)}$ sont tous égaux à partir d'un certain rang, c'est-à-dire que $k(n)$ est de la forme $k(n)=m(n)^{2}+i_{0}$. Ainsi, $a_{k(n)-i_{0}}=a_{m(n)^{2}}=m(n)$ tend vers l'infini, et les mots $\left(a_{k(n)} a_{k(n)-1} \ldots a_{1}\right)$ ne convergent pas.

Enfin, si une sous-suite $\left(a_{k(n)}\right)$ de quotients partiels de $\alpha$ tend vers l'infini, alors, $M$ étant fixé, $a_{k(n)}>M$ pour $n>n_{0}$, et les indices $k(n)$ sont de la forme $k(n)=m(n)^{2}+i$, avec $M \leq i \leq 2 m(n)+1$. Si $n_{1}$ est le plus grand entier tel que $k\left(n_{1}\right) \leq\left(m\left(n_{0}\right)+1\right)^{2}$, on a $k\left(n_{1}+1\right) \geq\left(m\left(n_{0}\right)+1\right)^{2}+M$, ce qui implique que $k\left(n_{1}+1\right)-k\left(n_{1}\right) \geq M$. Ainsi, $k(n)$ ne peut être à lacunes bornées, et $\alpha$ est bien un contre-exemple à une éventuelle réciproque.

On prouve aussi une condition suffisante mais non nécessaire d'existence d'une valeur d'adhérence sturmienne : un contre-exemple est donné par $\alpha=$ $[0 ; 1,1,2,1,2,1, \ldots, n, n-1, \ldots, 2,1, n, n-1, \ldots, 2,1, \ldots]$.

Corollaire 3.2. Si le développement en fraction continue de $\alpha$ contient des plages arbitrairement longues de quotients partiels bornés, les mots d'agencement des longueurs associés à $\alpha$ admettent une valeur d'adhérence sturmienne.

Preuve. Il existe alors une suite $k(n)$ de parité constante, et une autre suite $l(n)$ tendant vers l'infini, telles que $a_{p}<M$ pour $k(n) \leq p \leq k(n)+$ $l(n)$. Les mots $a_{k(n)+l(n)} \ldots a_{k(n)}$ étant dans le compact constitué des mots finis et infinis sur l'alphabet fini $\{1, \ldots, M\}$, il existe une suite extraite $(m(n))$ de $(k(n))$ telle que $\left(a_{m(n)+l(n)} \ldots a_{m(n)}\right)_{n>0}$ converge vers un mot $u$. 
Puisque $l(n)$ tend vers l'infini, les mots $a_{m(n)+l(n)} \ldots a_{1}$ convergent euxmême vers $u$, et le théorème 3.1 permet de conclure.

Introduisons deux nouvelles substitutions du monoïde de Sturm, qui complètent $\sigma_{1}$ et $\sigma_{2}$ :

$$
\begin{array}{ll}
\sigma_{3}(0)=01, & \sigma_{4}(0)=10, \\
\sigma_{3}(1)=1, & \sigma_{4}(1)=1 .
\end{array}
$$

Ces substitutions interviennent par exemple lorsqu'on recherche l'application de premier retour d'une rotation d'angle $\beta$ sur le plus grand des deux intervalles $] 1-\beta, 1]$ et $] \beta, 1]$ ou le plus grand de $[0, \beta[$ et $[0,1-\beta[$. On montre par induction qu'il s'agit d'un échange de deux intervalles, c'est-à-dire encore une rotation. On en déduit :

Proposition 3.2 (voir [2]). Soit $\beta$ un irrationnel et $\gamma$ défini par

$$
\gamma= \begin{cases}(2 \beta-1) / \beta & \text { si } \beta>1 / 2, \\ (1-\beta) / \beta & \text { si } \beta<1 / 2 .\end{cases}
$$

Soit $u_{\beta}$ et $u_{\gamma}$ (respectivement $v_{\beta}$ et $v_{\gamma}$ ) les codages à gauche (respectivement à droite) d'angle $\beta$ et $\gamma$ et d'intercept $1-\beta$ et $1-\gamma$. Alors

$$
\begin{array}{llll}
\text { si } \beta>1 / 2, & u_{\beta}=\sigma_{4}\left(u_{\gamma}\right) & \text { et } & v_{\beta}=\sigma_{3}\left(v_{\gamma}\right), \\
\text { si } \beta<1 / 2, & u_{\beta}=\sigma_{2}\left(u_{\gamma}\right) & \text { et } & v_{\beta}=\sigma_{1}\left(v_{\gamma}\right) .
\end{array}
$$

Corollaire 3.3. Si les $b_{n}$ sont non nuls, les mots $J_{b_{1}} I_{b_{2}} \ldots J_{b_{2 n+1}}(0)$ (respectivement $\left.I_{b_{1}} J_{b_{2}} \ldots J_{b_{2 n}}(0)\right)$ convergent vers le codage à gauche (respectivement à droite) d'angle $\beta=\left[0 ; 1, b_{1}, b_{2}, \ldots\right]$ et d'intercept $1-\beta$.

Preuve. En itérant la proposition 3.2, on obtient que les codages à gauche et à droite $u_{\alpha}$ et $v_{\alpha}$ d'angle $\alpha$ et d'intercept $1-\alpha$ sont liés au développement en fractions continues $\left[0 ; a_{1}, \ldots, a_{n}, \ldots\right]$ de $\alpha$ par les relations :

$$
\begin{aligned}
& u_{\alpha}=\lim \left(\sigma_{2}\right)^{a_{1}-1}\left(\sigma_{4}\right)^{a_{2}}\left(\sigma_{2}\right)^{a_{3}} \ldots\left(\sigma_{4}\right)^{a_{2 n}}(1), \\
& v_{\alpha}=\lim \left(\sigma_{1}\right)^{a_{1}-1}\left(\sigma_{3}\right)^{a_{2}}\left(\sigma_{1}\right)^{a_{3}} \ldots\left(\sigma_{1}\right)^{a_{2 n+1}}(0) .
\end{aligned}
$$

Les substitutions $I_{n}, J_{n}$ et $\sigma_{i}$ vérifient

$$
\sigma_{1}^{n}=E J_{n}, \quad \sigma_{2}^{n}=E I_{n}, \quad \sigma_{3}^{n}=I_{n} E, \quad \sigma_{4}^{n}=J_{n} E .
$$

On conclut en posant $a_{1}=1$ et $a_{n}=b_{n-1}$ pour $n>1$.

On en déduit une caractérisation des valeurs d'adhérence sturmiennes de la suite des mots d'agencement des longueurs.

ThÉORÈme 3.1. Les valeurs d'adhérence sturmiennes des mots d'agencement des longueurs associés à un angle $\alpha$ de convergents $p_{n} / q_{n}$ et de quotients partiels $a_{n}$ sont les codages de la trajectoire de $1-\beta$ par la rotation d'angle $\beta$ sur $[0,1[$, par rapport à la partition $[0,1-\beta[\cup[1-\beta, 1[$ 
(respectivement $] 0,1-\beta] \cup] 1-\beta, 1]$ ), ceci pour toutes les valeurs d'adhérence irrationnelles $\beta$ de la suite de nombres $\left[0 ; 1, a_{n}, \ldots, a_{1}\right]=q_{2 n} /\left(q_{2 n}+q_{2 n-1}\right)$ (respectivement $\left.q_{2 n+1} /\left(q_{2 n}+q_{2 n+1}\right)\right)$.

Preuve. Ceci découle directement de la proposition 3.1 et du corollaire 3.3 en remarquant que, par continuité du développement en fractions continues sur $\mathbb{R} \backslash \mathbb{Q}$, l'angle de la valeur d'adhérence sturmienne associée à une valeur d'adhérence $\left(b_{n}\right)$ des mots $a_{2 n} a_{2 n-1} \ldots a_{1}$ est la limite des rationnels $\left[0 ; 1, a_{2 n}, a_{2 n-1}, \ldots, a_{1}\right]=q_{2 n} /\left(q_{2 n-1}+q_{2 n}\right)$ (voir [8]).

Le fait que l'angle d'une valeur d'adhérence est toujours supérieur à $1 / 2$ est cohérent : en effet, par définition, l'image d'un mot par $Q_{n}$ contient beaucoup plus de 1 que de 0 . Ce théorème sera redémontré dans le cas quadratique de manière élémentaire dans la partie 4, et généralisé à d'autres mots d'agencement des longueurs dans la partie 5.

On déduit facilement, en terme de théorie des langages, que dès que le développement en fractions continues d'un angle contient toute suite finie d'entiers, le langage St des facteurs de tous les mots sturmiens peut se lire dans les mots d'agencement. De tels nombres forment un ensemble de mesure pleine par ergodicité de l'opérateur de décalage sur les fractions continues $(T(x)=\{1 / x\})$ par rapport à la mesure de Gauss, absolument continue par rapport à la mesure de Lebesgue (voir [5]).

Corollaire 3.4. Pour presque tout angle $\alpha$, toute suite équilibrée est valeur d'adhérence de la suite des mots d'agencement des longueurs. Pour de tels angles, l'ensemble des facteurs des mots d'agencement est égal à l'ensemble St des facteurs de tous les mots sturmiens.

4. Le cas quadratique. Si $\alpha$ est quadratique, le théorème de Lagrange (voir [8]) affirme que ses quotients partiels $a_{n}$ forment une suite ultimement périodique, et les théorèmes 2.1 et 3.1 nous disent que les valeurs d'adhérence des mots d'agencement sont toutes sturmiennes et en nombre fini. La proposition suivante montre que la réciproque est vraie.

Proposition 4.1. Les valeurs d'adhérence de la suite des mots d'agencement des longueurs associés à un angle $\alpha$ sont en nombre fini et toutes sturmiennes si et seulement si l'angle $\alpha$ est quadratique.

Preuve. Supposons que les mots d'agencement $\left(\Psi_{n}\right)$ admettent exactement $C$ valeurs d'adhérence qui sont toutes sturmiennes. Si la suite des quotients partiels $\left(a_{n}\right)$ n'était pas ultimement périodique de période $C$ !, il existerait une suite $\varphi(n)$ strictement croissante et de parité constante telle que $a_{\varphi(n)+C !} \neq a_{\varphi(n)}$ pour tout $n>0$. Posons $\varrho(n)=\varphi(n)+C+C$ !. Quitte à renommer $\varphi$, la compacité de $\{0,1\}^{\star}$ implique que $\left(\Psi_{\varrho(n)}\right)_{n>0}$ converge vers un mot $u$, qui est sturmien par hypothèse. 
Selon la proposition 3.1 , les mots $\left(a_{\varrho(n)} \ldots a_{1}\right)_{n>0}$ convergent vers un mot infini $v$, ce qui implique que chaque suite $\left(a_{\varrho(n)-p} \ldots a_{1}\right)_{n>0}$ converge elle-même vers $S^{p}(v)$, où $S$ est l'opérateur de décalage vers la droite. Puisque les valeurs d'adhérence sont en nombre fini, on en déduit qu'il existe $p_{0}<C$ et $T \leq C$ tels que $S^{p_{0}}(v)=S^{p_{0}+T}(v)$. L'application de $S$ à cette égalité implique alors que $S^{p}(v)=S^{p+C !}(v)$ pour tout $p \geq p_{0}$. En particulier, $S^{C}(v)=S^{C+C !}(v)$, c'est-à-dire, en reprenant la définition de $v$,

$$
\lim _{n \rightarrow \infty} a_{\varphi(n)+C !} \ldots a_{1}=\lim _{n \rightarrow \infty} a_{\varphi(n)} \ldots a_{1} .
$$

Ainsi, dès que $n$ est assez grand, $a_{\varphi(n)+C !}=a_{\varphi(n)}$, ce qui contredit le choix de $\varphi$.

Les valeurs d'adhérence sont alors identifiées :

Proposition 4.2. Si l'angle $\alpha$ est quadratique, les valeurs d'adhérence de la suite des mots d'agencement des longueurs sont points fixes de substitution.

Si $\alpha=\left[0 ; a_{1}, \ldots, a_{2 n_{0}},\left(c_{1}, \ldots, c_{2 T}\right)^{\infty}\right]$, les substitutions en question sont les $I_{c_{2 k}} J_{c_{2 k+1}} \ldots I_{c_{2 T}} J_{c_{1}} \ldots J_{c_{2 k-1}}$ et les $J_{c_{2 k+1}} I_{c_{2 k+2}} \ldots I_{c_{2 T}} J_{c_{1}} \ldots I_{c_{2 k}}$.

Preuve. Selon la proposition 3.1, une valeur d'adhérence $u$ des $\Psi_{n}$ commençant par 0 est limite de mots de la forme $I_{b_{1}} J_{b_{2}} \ldots J_{b_{2 n}}(0)$ où la suite $b=\left(b_{n}\right)$ est valeur d'adhérence des $a_{2 n} \ldots a_{1}$. Dans le cas qui nous concerne, $b$ est donc un mot périodique de période $2 T$. Or, pour tout $m, n$, le mot $I_{n} J_{m}(0)$ commence par 0 . Ainsi, si $\sigma$ est la substitution $I_{b_{1}} J_{b_{2}} \ldots J_{b_{2 T-1}}$, les mots $I_{b_{1}} J_{b_{2}} \ldots J_{b_{2 n}}(0)$ commencent tous par $\sigma^{[n / T]}(0)$. On déduit du fait que $\sigma(0)$ commence par 0 que $u$ est le point fixe de $\sigma$.

On retrouve ainsi de manière élémentaire le théorème 3.1 dans le cas quadratique.

Proposition 4.3. Soit $\alpha=\left[0 ; a_{1}, \ldots, a_{2 n_{0}},\left(c_{1}, \ldots, c_{2 T}\right)^{\infty}\right]$ un angle quadratique. Alors les valeurs d'adhérence des mots d'agencement des longueurs associés à $\alpha$ sont toutes sturmiennes et d'angles

$$
\alpha_{k}=\left[0 ; 1,\left(c_{k}, c_{k-1}, \ldots, c_{1}, c_{2 T}, \ldots, c_{k+1}\right)^{\infty}\right], \quad 0 \leq k<T .
$$

Preuve. Notons $\sigma_{k}$ la substitution $I_{c_{k}} J_{c_{k+1}} \ldots I_{c_{2 T}} J_{c_{1}} \ldots J_{c_{k-1}}$ si $k$ est pair et $J_{c_{k}} I_{c_{k+1}} \ldots I_{c_{2 T}} J_{c_{1}} \ldots I_{c_{k-1}}$ sinon. Soit $u_{k}$ le point fixe de $\sigma_{k}$, unique vu la définition des $J_{n}$. Soit $\alpha_{k}$ l'angle de $u_{k}$.

L'entier $k$ étant fixé, les mots $u_{k}$ et $u_{k+1}$ vérifient $u_{k+1}=\tau\left(u_{k}\right)$, avec $\tau \in$ $\left\{I_{c_{k+1}}, J_{c_{k+1}}\right\}$ suivant la parité de $k$. Notons $\beta_{n}$ et $\gamma_{n}$ (respectivement $\beta_{n}^{\prime}$ et $\gamma_{n}^{\prime}$ ) les nombres de 0 et 1 dans le début de $u_{k}$ de longueur $n$ (respectivement dans l'image de début par $\tau$, qui est un début de $u_{k+1}$ ). On a ainsi $\beta_{n}+\gamma_{n}$ $=n$. 
La définition géométrique des mots sturmiens impliquant que l'angle d'un mot sturmien $\left(v_{n}\right)$ est égal à la proportion de 1 dans ses préfixes, on a

$$
\alpha_{k}=\lim \frac{\gamma_{n}}{n} \quad \text { et } \quad \alpha_{k+1}=\lim \frac{\gamma_{n}^{\prime}}{\beta_{n}^{\prime}+\gamma_{n}^{\prime}} .
$$

Or, la définition de $\tau$ implique que $\beta_{n}^{\prime}=\gamma_{n}$ et $\gamma_{n}^{\prime}=\beta_{n}+c_{k+1} \gamma_{n}$. Ainsi

$$
\frac{1}{\alpha_{k+1}}-1=\lim _{n \rightarrow \infty} \frac{\gamma_{n}}{\beta_{n}+c_{k+1} \gamma_{n}}=\frac{1}{c_{k+1}+\frac{1}{\alpha_{k}}-1} .
$$

De proche en proche on obtient

$$
\frac{1}{\alpha_{k}}-1=\frac{1}{c_{k}+\frac{1}{\alpha_{k-1}}-1}=\ldots=\frac{1}{c_{k}+\frac{1}{c_{k-1}+\ldots+\frac{1}{c_{k+1}+\frac{1}{\alpha_{k}}-1}}}
$$

c'est-à-dire que

$$
\frac{1}{\alpha_{k}}-1=\left[0 ;\left(c_{k}, c_{k-1}, \ldots, c_{1}, c_{2 T}, \ldots, c_{k+1}\right)^{\infty}\right] .
$$

5. Autres mots d'agencement des longueurs. Le théorème des trois longueurs affirme en particulier que seules deux longueurs apparaissent sur le cercle si et seulement si le nombre de points placés sur le cercle est un convergent partiel de l'angle. On définit ainsi un mot d'agencement des longueurs $\Phi_{n, i}$ pour tout convergent $q_{n, i}$, avec $i \leq a_{n}$. On a alors $\Psi_{n}=\Phi_{n+1,1}$.

Chaque mot $\Phi_{n, i}$ étant l'image de $\Psi_{n-1}$ par une substitution du monoïde de Sturm ne dépendant que de $i$ (voir [14]), les suites de mots $\left(\Phi_{n, i_{0}}\right)$, pour $i_{0}$ fixé, ont des propriétés identiques à celles qui ont été prouvées pour $\left(\Psi_{n}\right)$. Seule la valeur des angles des valeurs d'adhérence sturmiennes est modifiée : leur développement en fraction continue ne commence plus par $[0 ; 1]$ mais par $\left[0 ; i_{0}\right]$. Par exemple, on peut s'intéresser comme dans [15] à la subdivision du cercle par $q_{n}$ points, c'est-à-dire aux mots $\Phi_{n, a_{n}}$.

Proposition 5.1. Les mots d'agencement $\Phi_{n, a_{n}}$, obtenus en découpant le cercle par les $\{n \alpha\}$ pour $0 \leq n<q_{n}$, ont pour valeurs d'adhérence sturmiennes les codages sur $[0,1-\beta[\cup[1-\beta, 1[$ (respectivement $] 0,1-\beta] \cup$ $11-\beta, 1])$ de la trajectoire de $1-\beta$ par la rotation d'angle $\beta$, ceci pour toute valeur d'adhérence irrationnelle $\beta$ des nombres $q_{2 n-1} / q_{2 n}$ (respectivement $\left.q_{2 n} / q_{2 n+1}\right)$, où les $q_{n}$ sont les convergents de $\alpha$.

Preuve. Selon [14] les mots d'agencement sont tels que $\Psi_{n}=I_{1}\left(\Phi_{n, a_{n}}\right)$ si $n$ est pair et $\Psi_{n}=J_{1}\left(\Phi_{n, a_{n}}\right)$ sinon. Si $v$ est une valeur d'adhérence sturmienne de $\left(\Phi_{n, a_{n}}\right)$ qui s'écrit $v=\lim _{n \rightarrow \infty} \Phi_{k(n), a_{k(n)}}$ avec $k(n)$ pair, la suite 
$\left(\Psi_{k(n)}\right)$ converge vers $I_{1}(v)$. Selon la proposition 3.1, il existe des entiers $b_{i}$ tels que

$$
\begin{aligned}
I_{1}(v) & =\lim _{n \rightarrow \infty} I_{b_{1}} J_{b_{2}} \ldots J_{b_{2 n}}(0)=\lim _{n \rightarrow \infty} E \sigma_{2}^{b_{1}} \sigma_{4} E^{b_{2}} \ldots \sigma_{4} E^{b_{2 n}}(0) \\
& =\lim _{n \rightarrow \infty} E \sigma_{2}^{b_{1}} \sigma_{4}^{b_{2}} \ldots \sigma_{4}^{b_{2 n}}(1) .
\end{aligned}
$$

Puisque par définition $I_{1}=E \sigma_{2}$, on obtient ainsi que $v$ est la limite des $\sigma_{2}^{b_{1}-1} \sigma_{4}^{b_{2}} \ldots \sigma_{4}^{b_{2 n}}(1)$, c'est-à-dire l'extension gauche du mot caractéristique d'angle $\left[0 ; b_{1}, b_{2}, \ldots\right]=\left[0 ; a_{k(n)}, a_{k(n)}-1, \ldots, a_{1}\right]=q_{k(n)-1} / q_{k(n)}$.

Dans le cas particulier où $\alpha$ est de la forme $\alpha=[0 ; a, a, \ldots]$, la proposition 5.1 est une version plus faible d'un résultat de van Ravenstein et al. [15], qui dit que pour de tels angles, le mot $\Phi_{n, a_{n}}$ privé de sa première et de sa dernière lettre est un préfixe du mot sturmien caractéristique d'angle $\alpha$. On remarque que ceci implique bien que les suites $\left(\Phi_{2 n, a_{2 n}}\right)$ et $\left(\Phi_{2 n+1, a_{2 n+1}}\right)$ convergent vers les codages à droite et à gauche de la rotation d'angle $\alpha$ et d'intercept $1-\alpha$.

Van Ravenstein et al. concluent [15] en demandant quels sont les angles qui vérifient une propriété identique à celle qu'il établit pour les $[0 ; a, a, \ldots]$. La réponse à cette question se déduit de la proposition 5.1.

Proposition 5.2. Soit $\alpha$ un angle tel que les mots d'agencement des longueurs $\Phi_{n, a_{n}}$ privés de leur première et dernière lettre sont des débuts du mot caractéristique d'angle $\alpha$. Alors le développement en fraction continue de $\alpha$ est de la forme $[0 ; a, a, \ldots]$.

Preuve. Soit $\alpha$ vérifiant les hypothèses ci-dessus. La suite des mots d'agencement $\left(\Phi_{n, a_{n}}\right)$ admet alors exactement deux valeurs d'adhérence : les prolongements à gauches par 0 et $1 \mathrm{du}$ mot caractéristique d'angle $\alpha$. Selon la proposition 4.1, l'angle $\alpha$ est donc quadratique. De plus, les valeurs d'adhérence sont de même angle, à savoir $\alpha$. Selon la proposition 5.1 , le développement en fraction continue de $\alpha$ ne peut donc être qu'ultimement constant, égal à un entier $a$. La proposition 5.1 implique alors que l'angle des valeurs d'adhérence est $\alpha_{1}=[0 ; a, a, \ldots]$, qui doit être égal à $\alpha$.

\section{Références}

[1] P. Alessandri and V. Berthé, Three distance theorems and combinatorics on words, Enseign. Math. (2) 44 (1998), 103-132.

[2] P. Arnoux, S. Ferenczi and P. Hubert, Trajectories of rotations, Acta Arith. 87 (1999), 209-217.

[3] J. Berstel, Recent results on sturmian words, in: Developments in Language Theory II, World Sci., 1996, 13-24.

[4] V. Berthé, Fréquences des facteurs des suites sturmiennes, Theoret. Comput. Sci. 165 (1996), 295-309. 
[5] P. Billingsley, Ergodic Theory and Information, Wiley, New York, 1965.

[6] J. Cassaigne, Limit values of the recurrence quotient for Sturmian sequences, in: Words (Rouen, 1997), Theoret. Comput. Sci. 218 (1999), 3-12.

[7] D. Crisp, W. Moran, A. Pollington and P. Shiue, Substitution invariant cutting sequences, J. Théor. Nombres Bordeaux 5 (1993), 123-137.

[8] G. H. Hardy and E. M. Wright, An Introduction to the Theory of Numbers, Oxford Science Publications, 1979.

[9] M. Morse and G. A. Hedlund, Symbolic dynamics II. Sturmian trajectories, Amer. J. Math. 60 (1940), 1-42.

[10] G. Rauzy, Echanges d'intervalles et transformations induites, Acta Arith. 34 (1979), 315-328.

[11] P. Séébold, On the conjugation of standard morphisms, Theoret. Comput. Sci. 195 (1998), 91-109.

[12] N. B. Slater, Gaps and steps for the sequence $n \theta \bmod 1$, Proc. Cambridge Philos. Soc. 63 (1967), 1115-1123.

[13] S. Świerczkowski, On successive settings of an arc on the circumference of a circle, Fund. Math. 46 (1958), 187-189.

[14] T. van Ravenstein, The three gap theorem (Steinhaus conjecture), J. Austral. Math. Soc. Ser. A 45 (1988), 360-370.

[15] T. van Ravenstein, G. Winley and K. Tognetti, Characteristics and the three gap theorem, Fibonacci Quart. 28 (1990), 204-214.

Institut de Mathématiques de Luminy

CNRS-UPR 9016, Case 907

163 avenue de Luminy

13288 Marseille Cedex 9, France

E-mail: siegel@iml.univ-mrs.fr 